\title{
A dipole feeder for circularly and linearly polarized cross shape loop/spiral antennas
}

\author{
Mayumi Matsunaga ${ }^{\text {a) }}$ \\ Department of Electrical and Electronic Engineering, Ehime University, \\ 3 Bunkyo-cho, Matsuyama, Ehime 790-8577, Japan \\ a)mmayumi@m.ieice.org
}

Abstract: A novel feeding way for balanced feed antennas is presented. To construct the balanced feed antenna, a cross shape loop/spiral antenna (CSA) - a CP antenna invented by the author - needs to be fed through a well-balanced port. A poor current balance will bring a serious deterioration in $\mathrm{CP}$ radiation. In addition, the current balance of general ports is not adequate. For these reasons, a feeder reducing the influence of an unbalanced current should be suggested. In this paper, a dipole is employed as a feeder for CSA. $\mathrm{S}_{11}$ characteristics and $\mathrm{CP}$ radiation patterns obtained by being fed through a coaxial cable (an unbalanced feed) are compared with those obtained by being fed through a balanced port to discuss the effectiveness of the dipole feeder.

Keywords: circularly polarized antennas, dipole feeders, baluns

Classification: Microwave and millimeter-wave devices, circuits, and modules

\section{References}

[1] H. Wong, et al.: "Small antennas in wireless communications," Proc. IEEE 100 (2012) 2109 (DOI: 10.1109/JPROC.2012.2188089).

[2] G. Augustin and T. A. Denidni: "Coplanar waveguide-fed uniplanar trapezoidal antenna with linear and circular polarization," IEEE Trans. Antennas Propag. 60 (2012) 2522 (DOI: 10.1109/TAP.2012.2189724).

[3] A. A. Gheethan and D. E. Anagnostou: "Broadband and dual-band coplanar folded-slot antennas (CFSAs)," IEEE Antennas Propag. Mag. 53 (2011) 80 (DOI: 10.1109/MAP.2011.5773572).

[4] R. Li, et al.: "Broadband circularly polarized rectangular loop antenna with impedance matching," IEEE Microw. Wireless Compon. Lett. 16 (2006) 52 (DOI: 10.1109/LMWC.2005.859946).

[5] H. Nakano, et al.: "A low-profile conical beam loop antenna with an electromagnetically coupled feed system," IEEE Trans. Antennas Propag. 48 (2000) 1864 (DOI: 10.1109/8.901276).

[6] M. Matsunaga: "A linearly and circularly polarized double-band cross spiral antenna,” IEICE Trans. Commun. E99-B (2016) 430 (DOI: 10.1587/transcom. 2015EBP3222).

[7] T. Fukasawa, et al.: "Extended S-parameter method including radiation pattern measurements of an antenna," IEEE Trans. Antennas Propag. 60 (2012) 5645 (DOI: 10.1109/TAP.2012.2210181).

[8] R. Lampe: "Design formulas for an asymmetric coplanar strip folded dipole," 
IEEE Trans. Antennas Propag. 33 (1985) 1028 (DOI: 10.1109/TAP.1985. 1143698).

[9] M. Matsunaga: "A linearly and circularly polarized triple-band cross spiral antenna fed by a dipole," IEICE Trans. Electron. (Japanese Edition) J98-C (2015) 329.

[10] M. Matsunaga: "Effectiveness of a dipole feeder for the cross spiral antenna which is a circularly and linearly polarized planar antenna," Proc. Int. Symp. Antennas Propag. (2015) 549.

\section{Introduction}

A balanced feed antenna is an attractive built-in antenna whose configuration is so compact and low-profile as to be easily integrated in RF circuits [1]. In addition, it is well known that a balanced feed antenna (loop antennas and dipole antennas) can constitute a compact and low-profile circularly polarized (CP) antenna [2, 3, 4, $5,6]$. For these balanced feed $\mathrm{CP}$ antennas, the balance of the current at feed ports is very important. This means that $\mathrm{CP}$ characteristics will be deteriorated if the balance of the current is fair. The current balance of general RF circuits, however, is not always equal [7]. Therefore, a feeder, which can neutralize unbalanced currents, is an essential key for firm characteristics of antennas.

The author invented a compact and low-profile loop/spiral antenna, CSA, radiating $\mathrm{CP}$ wave [6]. CSA radiates $\mathrm{CP}$ waves by using a transmission line mode [8] of a folded dipole antenna or a quad loop antenna for constituting rotating current flows on antenna elements. Therefore CSA will not radiate good CP waves if the current balance of its port is poor.

In conventional studies $[2,3,4,5]$, many $\mathrm{CP}$ antennas employ microwave balun circuits to be fed well-balanced currents. A coplanar waveguide is used for realizing the balanced feed in [2,3], and microstrip integrated balun is joined vertically to the antenna in [4]. These baluns surely help to transform unbalanced currents into balanced currents. However, the size of these microwave baluns is the almost same size as the antennas; this leads to an increase of antenna sizes. On the other hand, a monopole is used as a feeder, which is close to a loop antenna in [5]. This solution is employed in many publications because it is an easy way to feed a loop antenna through an unbalanced port. A feeder, which is a compact integrated element of CSA and reduces the effect of unbalanced currents, is presented in this paper.

A dipole - not a monopole - is employed as a feeder in this paper. This is because the author wants to make multi-polarized antenna by means of using the dipole not only as a feeder but also as a radiating element of linear polarization (LP) [9]. CSA will become the antenna radiating CP simultaneously with LP if a dipole feeder works as a radiating element. In this paper, the effectiveness of the dipole feeder for CSA is discussed by comparing the measurement characteristics of CSA fed directly and CSA fed indirectly through a dipole feeder (DF-CSA) [10].

In section 2, the configuration of the CSA fed through a dipole feeder, DF- 
simulation results obtained with a balanced feed are compared with the simulation results obtained with an unbalanced feed and the measurement results obtained with a coaxial cable.

\section{Structures of CSA}

In this section, the structure of a basic CSA and the structure of the CSA fed by a dipole (DF-CSA) are explained. Fig. 1(a) shows a basic structure of CSA [6]. The basic CSA is constituted by a cross-shaped single loop, and is fed with a balanced port. Fig. 1(b) shows the structure of DF-CSA [9]. A dipole element is put in the center of a basic CSA. The dipole element is fed through via holes in the dipole element. The spiral element is a parasitic one, fed indirectly by the dipole element. Two gaps are made on the spiral element to help a dipole and a spiral work as they are intended. This means that electromagnetic (EM) interference happens when the dipole and the spiral are close to each other; the two gaps eliminate the EM interference.

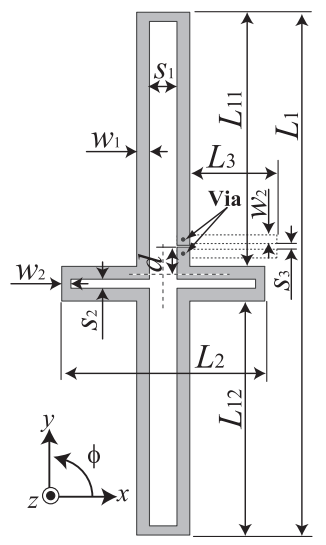

(a) A structure of a basic CSA

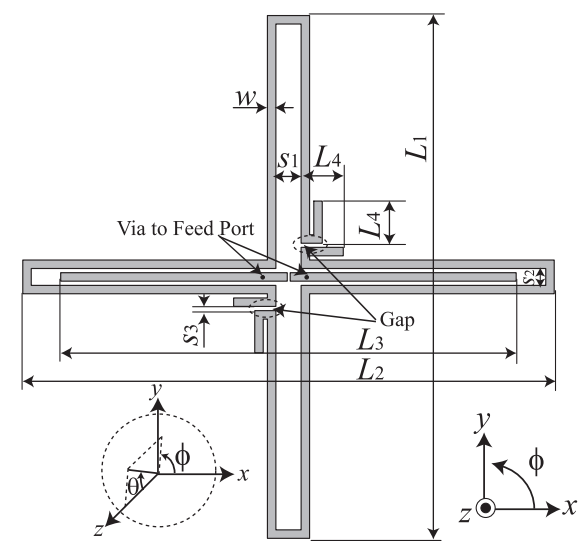

(b) A Structure of DF-CSA

Fig. 1. Structures of (a) a basic CSA and (b) a DF-CSA.

\section{Discussion of CSA characteristics}

In this section, the effectiveness of a dipole feeder is discussed by comparing $\mathrm{S}_{11}$ characteristics and radiation patterns of a basic CSA with those of DF-CSA. The results shown in this section are obtained when the basic CSA and DF-CSA are formed by using the measurements shown in Table I and the PCB whose $\varepsilon_{\mathrm{r}}$ is 4.4, $\tan \delta$ is 0.016 and thickness is $1.6 \mathrm{~mm}$.

Table I. Dimensions of CSA: (a) Basic CSA, (b) DF-CSA.

(a) Basic CSA

\begin{tabular}{c|c|c|c|c|c|c|c|c|c|c}
\hline $\boldsymbol{L}_{\mathbf{1}}$ & $\boldsymbol{L}_{\mathbf{1 1}}$ & $\boldsymbol{L}_{\mathbf{1 2}}$ & $\boldsymbol{L}_{\mathbf{2}}$ & $\boldsymbol{L}_{\mathbf{3}}$ & $\boldsymbol{s}_{\mathbf{1}}$ & $\boldsymbol{s}_{\mathbf{2}}$ & $\boldsymbol{s}_{\mathbf{3}}$ & $\boldsymbol{w}_{\mathbf{1}}$ & $\boldsymbol{w}_{\mathbf{2}}$ & $\boldsymbol{d}$ \\
\hline 57.2 & 27.8 & 25.6 & 22.2 & 9.6 & 3.0 & 1.0 & 0.6 & 1.4 & 1.0 & 3.0 \\
\hline
\end{tabular}

(b) DF-CSA

\begin{tabular}{c|c|c|c|c|c|c|c}
\hline $\boldsymbol{L}_{\mathbf{1}}$ & $\boldsymbol{L}_{\mathbf{2}}$ & $\boldsymbol{L}_{\mathbf{3}}$ & $\boldsymbol{L}_{\mathbf{4}}$ & $\boldsymbol{s}_{\mathbf{1}}$ & $\boldsymbol{s}_{\mathbf{2}}$ & $\boldsymbol{s}_{\mathbf{3}}$ & $\boldsymbol{w}$ \\
\hline 59.8 & 60.8 & 52.2 & 5.0 & 3.0 & 2.0 & 0.3 & 1.0 \\
\hline
\end{tabular}

First, $\mathrm{S}_{11}$ characteristics and radiation patterns of a basic CSA fed through a balanced port are compared with those of a basic CSA fed through an unbalanced 
port to understand how the basic CSA is affected by an unbalanced feed. Fig. 2 shows the $S_{11}$ characteristics of the basic CSA. The simulation results with a balanced port are very different from the simulation results with an unbalanced port. In addition, the measurement results with a coaxial cable are similar to the simulation results with an unbalanced feed. Therefore, the $S_{11}$ characteristics change when it is fed through an unbalanced port; the measurement results contain the effect of the unbalanced feed.

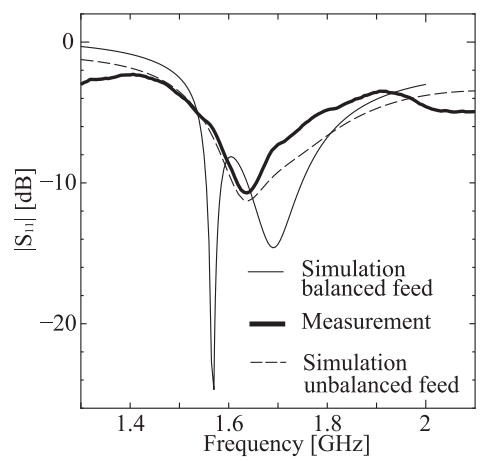

Fig. 2. $\mathrm{S}_{11}$ characteristics of a basic CSA, comparing the results obtained by a balanced feed with those obtained by an unbalanced feed.

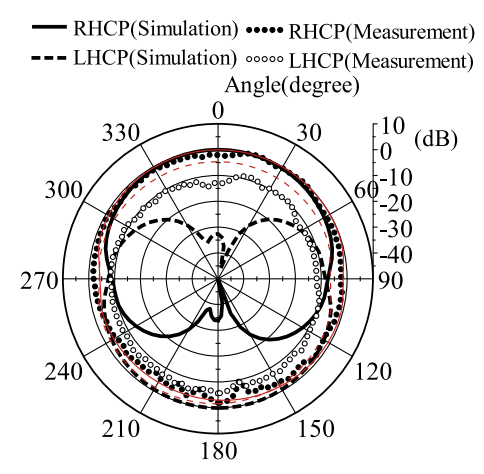

(a) $\phi=0^{\circ}$ cut at $1.57 \mathrm{GHz}$

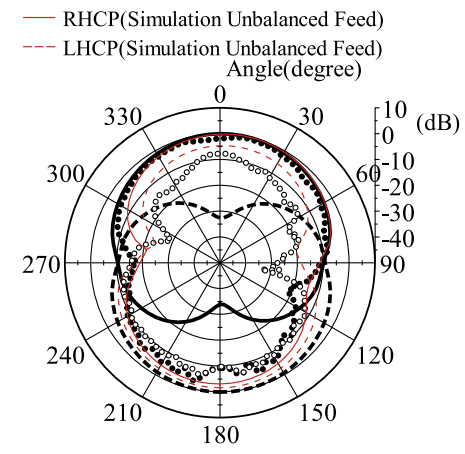

(b) $\phi=90^{\circ}$ cut at $1.57 \mathrm{GHz}$

Fig. 3. CP radiation patterns in (a) $\phi=0^{\circ}$ cut and in (b) $\phi=90^{\circ}$ cut at $1.57 \mathrm{GHz}$ of a basic CSA. The results obtained by a balanced feed are compared with those obtained by an unbalanced feed.

Fig. 3 shows CP radiation patterns at $1.57 \mathrm{GHz}$ in (a) the $\phi=0^{\circ}$ cut plane and (b) the $\phi=90^{\circ}$ cut plane. Note that the radiation gains are normalized by the maximum gain in the radiation patterns. The simulation results with a balanced feed are very different from the simulation results with an unbalanced feed. That is to say good right-handed CP (RHCP) is radiated in the simulation results with a balanced feed, however, the difference between RHCP and left-handed CP (LHCP) is small in the simulation results with an unbalanced feed. The measurement results show that the basic CSA will not radiate good CP waves anymore because of the unbalanced currents when it is fed by an unbalanced feed.

Second, $\mathrm{S}_{11}$ characteristics and radiation patterns of DF-CSA fed through a balanced port are compared with those of DF-CSA fed through an unbalanced port to understand the effects of a dipole feeder on unbalanced currents. Fig. 4 and 5 respectively show $S_{11}$ characteristics and radiation patterns of DF-CSA. Overall, 


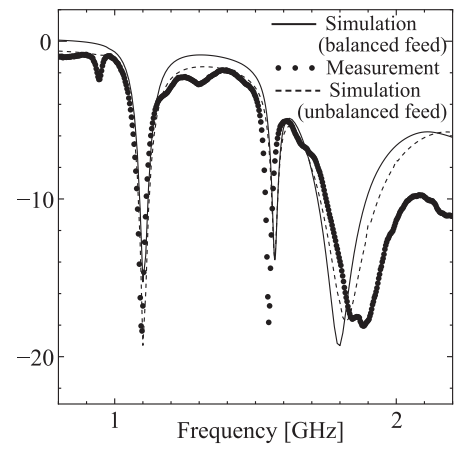

Fig. 4. $\mathrm{S}_{11}$ characteristics of a DF-CSA. The results obtained by a balanced feed are compared with those obtained by an unbalanced feed.

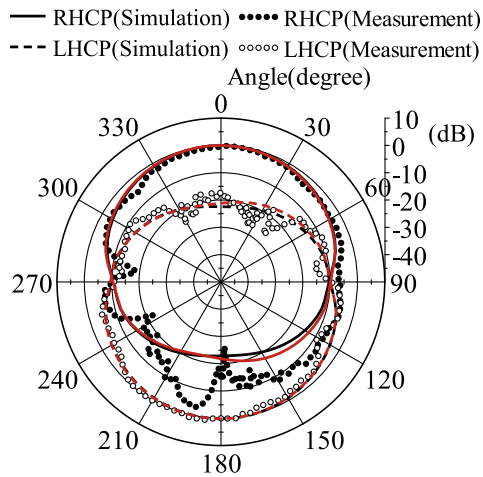

(a) $\phi=0^{\circ}$ cut at $1.57 \mathrm{GHz}$

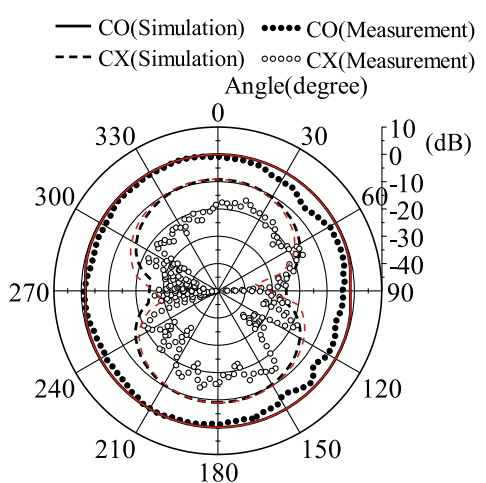

(c) $\phi=0^{\circ}$ cut at $1.1 \mathrm{GHz}$

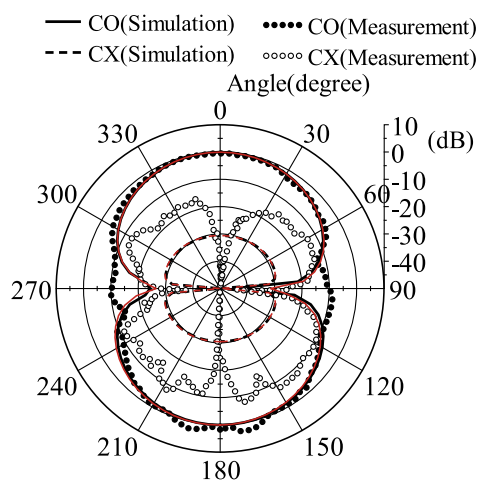

(e) $\phi=0^{\circ}$ cut at $1.8 \mathrm{GHz}$

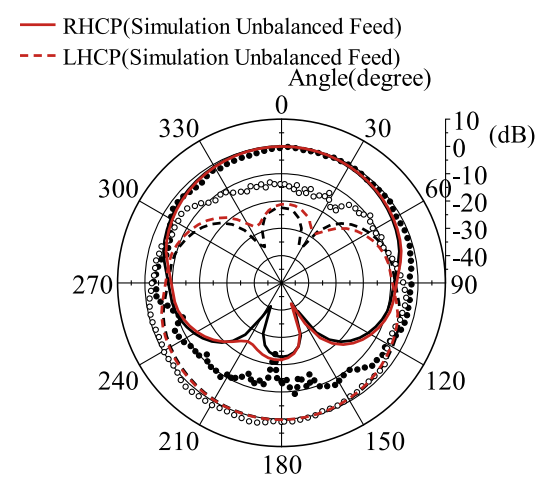

(b) $\phi=90^{\circ}$ cut at $1.57 \mathrm{GHz}$

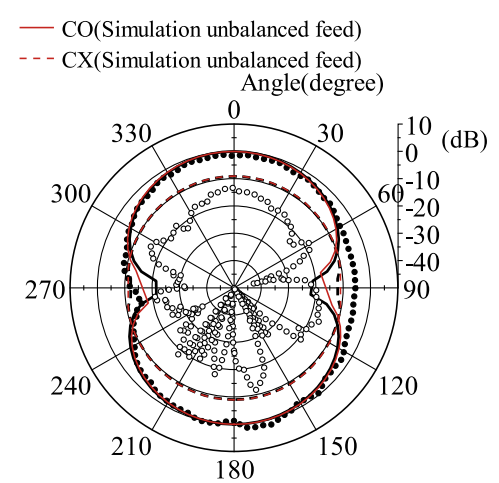

(d) $\phi=90^{\circ}$ cut at $1.1 \mathrm{GHz}$

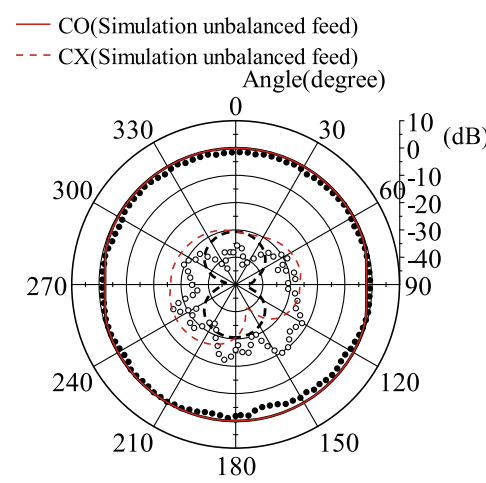

(f) $\phi=90^{\circ}$ cutat $1.8 \mathrm{GHz}$ 
the simulation results with a balanced port are similar to not only the simulation results with an unbalanced port but also the measurement results with a coaxial cable. This means that the unbalanced feed has little effect on $\mathrm{S}_{11}$ characteristics and radiation patterns of DF-CSA.

Discussing the results in more detail, in measurement results of $\mathrm{CP}$ waves at 1.57 GHz, $\mathrm{S}_{11}$ shifts to a bit lower frequency; LHCP radiations (Fig. 5(a) and 5(b)) tilt about five degrees toward the dipole's negative pole element. This can be explained that CSA's left and upper elements are fed through the dipole's left element indirectly and CSA's right and lower elements are fed through the dipole's right element indirectly. The current amplitudes on the left and the right of the dipole element are different, when the center line and the outer conductor of a coaxial cable connect into the left and the right of the dipole element, respectively. For this reason, the radiation directivity of the DF-CSA rotates some angles toward the direction of the right dipole element as shown in Fig. 5(a) and (b). However, the DF-CSA radiates good circular polarization, because there is no difference between the current phases of left and right dipole elements. According to the measurement result in Fig. 5(a) and (b), rotation angle of the radiation directivity due to the current amplitude difference is about five degrees. This means that the dipole feeder helps to reduce the effect of unbalanced feed.

In measurement results of LP waves at $1.1 \mathrm{GHz}$, because DF-CSA radiates LP waves by using a standing-wave mode, there are few effects of unbalanced feed in $\mathrm{S}_{11}$ and radiation patterns (Fig. 5(c) and 5(d)). In measurement results of LP waves at $1.8 \mathrm{GHz}, \mathrm{S}_{11}$ is affected by the unbalanced feed due to the dipole mainly works for radiating; the sideways radiation of the cross-polarization in the $\phi=90^{\circ}$ cut (Fig. 5(f)) becomes a bit higher than simulation results with a balanced feed. This means that the dipole feeder does not work as a balun at $1.8 \mathrm{GHz}$, because the dipole itself is a radiation element and it is fed directly with an unbalanced feed.

These results show that $\mathrm{S}_{11}$ characteristics and CP radiations of DF-CSA are stable because of the dipole feeder even if DF-CSA is fed by an unbalanced feed, although those of a basic CSA are very affected by an unbalanced feed. In conclusion, the dipole feeder reduces the effect of an unbalanced feed on CSA.

\section{Conclusion}

A dipole feeder that can reduce the effect of unbalanced currents on CP radiation of CSA is presented. The $\mathrm{S}_{11}$ and radiation patterns of the basic CSA show that the basic CSA is greatly affected by the unbalanced currents. On the other hand, the $\mathrm{S}_{11}$ and radiation patterns of DF-CSA show that the dipole feeder helps DF-CSA not to be affected by unbalanced currents. Therefore, the dipole feeder made it possible for the CSA to radiate good LP simultaneously with CP. Moreover the dipole feeder works as a kind of balun, helping to also reduce unbalanced currents. In conclusion, CSA became a multi-polarized antenna that is very simple, planar and a balanced feed as to be easily integrated into RF circuits of mobile devices.

\section{Acknowledgments}

This work was supported by JSPS KAKENHI Grant Numbers 26420359 and $15 \mathrm{H} 02135$. 